FACTA UNIVERSITATIS (NIŠ)

Ser. Math. Inform. Vol. 35, No 5 (2020), 1461-1474

https://doi.org/10.22190/FUMI2005461G

\title{
NEW SUBCLASS OF MEROMORPHIC FUNCTIONS BY THE GENERALIZATION OF THE $q$-DERIVATIVE OPERATOR
}

\author{
Mohammad Hassn Golmohammadi, Shahram Najafzadeh \\ and Mohammad Reza Foroutan
}

(C) 2020 by University of Niš, Serbia | Creative Commons Licence: CC BY-NC-ND

Abstract. In this paper, we introduce a new subclass of meromorphic functions, using the exponent $q$-derivative operator. Afterwards, coefficient estimates, extreme points, convex linear combination, radii of starlikeness and convexity and finally partial sum property have been investigated.

Keywords: Meromorphic functions; $q$-derivative; coefficient bound; extreme point; convex set; Hadamard product.

\section{Introduction}

Fractional calculus have started to appear more and more frequently for the modelling of relevant systems in several fields of applied sciences. For more details, one may refer to the books $[6,7,9]$ and the recent papers on the subject. The theory of $q$-analysis has attracted a considerable effort of researches due to its application in many branches of mathematics and physics and $q$-theory has an important role in various branches of mathematics and physics as for example, in the areas of special functions, ordinary fractional calculus, optimal control problems, $q$-difference, $q$-integral equations, $q$-transform analysis and in quantum physics (see for instance, $[1,2,3,4,5,8,10,16])$.

The theory of univalent functions can be described by using the theory of the $q$ calculus. Moreover, in recent years, such $q$-calculus as the $q$-integral and $q$-derivative have been used to construct several subclasses of analytic functions (see, for example, $[12,13,14,15,17])$.

Let $\Sigma$ denote the class of meromorphic functions of the form

$$
f(z)=\frac{1}{z}+\sum_{k=1}^{+\infty} a_{k} z^{k-1}
$$

Received February 15, 2020; accepted June 10, 2020

2020 Mathematics Subject Classification. Primary 20D15; Secondary 20F14 
which are analytic in the punctured unit disk

$$
\triangle^{*}=\{z \in \mathbb{C}: 0<|z|<1\} .
$$

Gasper and Rahman [7] defined the $q$ - derivative of a function $f(z)$ of the form equation 1.1 by

$$
D_{q} f(z)=\frac{f(q z)-f(z)}{(q-1) z} .
$$

where $z \in \triangle^{*}$ and $0<q<1$.

Therefore, the $q$ - derivative of $f(z)=z^{k-1}$ is given by

$$
D_{q} z^{k-1}=\frac{(z q)^{k-1}-z^{k-1}}{(q-1) z}=[k-1]_{q} z^{k-2}
$$

Our aim in this paper is to introduce a new operator and a new class of functions given by equation 1.1. So we have

$$
\begin{gathered}
D_{q}^{n} f(z)=\frac{(-1)^{n} \prod_{k=1}^{n}\left(1+q+q^{2}+\cdots+q^{k-1}\right)}{q^{\left(\begin{array}{c}
n+1 \\
n-1
\end{array}\right)} z^{n+1}}+\sum_{k=1}^{\infty} \prod_{i=1}^{n}[k-i]_{q} a_{k} z^{k-n-1} \\
\left(z \in \triangle^{*}, n \in \mathbb{N}=\{1,2, \cdots\}\right)
\end{gathered}
$$

where

$$
\prod_{k=1}^{n}\left(1+q+q^{2}+\cdots+q^{k-1}\right)=(1+q)\left(1+q+q^{2}\right) \cdots\left(1+q+\cdots+q^{k-1}\right)
$$

and

$$
\prod_{i=1}^{n}[k-i]_{q}:=\left(\frac{1-q^{k-1}}{1-q}\right)\left(\frac{1-q^{k-2}}{1-q}\right) \cdots\left(\frac{1-q^{k-n}}{1-q}\right) .
$$

also $\prod_{i=1}^{n}[k-i]_{q} \rightarrow \prod_{i=1}^{n}(k-i)$ as $q \rightarrow \overline{1}$. So we conclude

$$
\lim _{q \rightarrow \overline{1}} D_{q}^{n} f(z)=f^{(n)}(z) \quad, \quad z \in \triangle^{*},
$$

see also [11].

For $n \in \mathbb{N}, 0<q<1,0 \leq \lambda \leq 1,0<\alpha \leq 1$ and $\beta>0$, let $\sum_{q}(n ; \lambda, \alpha, \beta)$ be the subclass of $\sum$ consisting of functions $f$ of the form equation 1.1 and satisfying the condition

$$
\begin{aligned}
& \left|\frac{z^{n+3}\left(D_{q}^{n} f(z)\right)^{\prime \prime}+z^{n+2}\left(D_{q}^{n} f(z)\right)^{\prime}-\frac{(-1)^{n}(n+1)^{2}}{q^{\left(\begin{array}{c}
n+1 \\
n-1
\end{array}\right)}} \prod_{k=1}^{n}\left(1+q+\cdots+q^{k-1}\right)}{\lambda z^{n+1}\left(D_{q}^{n} f(z)\right)+\frac{(-1)^{n} \prod_{k=1}^{n}\left(1+q+\cdots+q^{k-1}\right)}{q^{\left(\begin{array}{c}
n+1 \\
n-1
\end{array}\right)}}+\frac{(1+\lambda) \alpha}{q^{\left(\begin{array}{c}
n+1 \\
n-1
\end{array}\right)}} \mid}\right| \\
& <\beta .
\end{aligned}
$$

We also derive some results given various coefficient inequalities, Radii condition and Hadamard product. 


\section{Main Results}

Unless otherwise mentioned, we suppose throughout this paper that $n \in \mathbb{N}$, $0<q<1,0 \leq \lambda<1,0<\alpha<1$ and $\beta>0$. First we state coefficient estimates on the class $\sum_{q}(n ; \lambda, \alpha, \beta)$.

Theorem 2.1. Let $f(z) \in \sum$, then $f(z) \in \sum_{q}(n ; \lambda, \alpha, \beta)$ is and only if

$$
\begin{aligned}
& \sum_{k=1}^{+\infty} \prod_{i=1}^{n}[k-i]_{q}\left((k-n-1)^{2}+\lambda \beta\right) a_{k} \\
\leq & \frac{\beta(1+\lambda)\left((-1)^{n-1} \prod_{k=1}^{n}\left(1+q+q^{2} \cdots+q^{k-1}\right)-\alpha\right)}{q^{\left(\begin{array}{l}
n+1 \\
n-1
\end{array}\right)}} .
\end{aligned}
$$

Proof. Let $f(z) \in \sum_{q}(n ; \lambda, \alpha, \beta)$, then equation 1.6 holds true. So by replacing equation 1.3 in equation 1.6 we have

$$
\begin{aligned}
& \mid \frac{\left.\sum_{k=1}^{+\infty}\left(\prod_{i=1}^{n}[k-i]_{q}(k-n-1)(k-n-2)+\prod_{i=1}^{n}[k-i]_{q}(k-n-1)\right) a_{k} z^{k}\right)}{\frac{(1+\lambda)}{q^{\left(\begin{array}{c}
n+1 \\
n-1
\end{array}\right)}(-1)^{n}} \prod_{k=1}^{n}\left(1+q+\cdots+q^{k-1}\right)+\lambda \sum_{k=1}^{\infty} \prod_{i=1}^{n}[k-i]_{q} a_{k} z^{k}+\frac{(1+\lambda) \alpha}{q^{\left(\begin{array}{c}
n+1 \\
n-1
\end{array}\right)}} \mid} \\
& <\beta .
\end{aligned}
$$

or

$$
\begin{aligned}
& \left|\frac{\sum_{k=1}^{+\infty} \prod_{i=1}^{n}[k-i]_{q}(k-n-1)^{2} a_{k} z^{k}}{\frac{(1+\lambda)}{q^{\left(\begin{array}{c}
n+1 \\
n-1
\end{array}\right)}}\left((-1)^{n-1} \prod_{k=1}^{n}\left(1+q+\cdots+q^{k-1}\right)-\alpha\right)-\lambda \sum_{k=1}^{+\infty} \prod_{i=1}^{n}[k-i]_{q} a_{z} z^{k}}\right| \\
& <\beta .
\end{aligned}
$$

Since $\operatorname{Re}(z) \leq|z|$ for all $z$, therefore

$$
\begin{aligned}
& \operatorname{Re}\left\{\frac{\sum_{k=1}^{+\infty} \prod_{i=1}^{n}[k-i]_{q}(k-n-1)^{2} a_{k} z^{k}}{\left.\frac{(1+\lambda)}{q^{\left(\begin{array}{c}
n+1 \\
n-1
\end{array}\right)}\left((-1)^{n-1} \prod_{k=1}^{n}\left(1+q+\cdots+q^{k-1}\right)-\alpha\right)-\lambda \sum_{k=1}^{+\infty} \prod_{i=1}^{n}[k-i]_{q} a_{z} z^{k}}\right\}}\right. \\
& <\beta .
\end{aligned}
$$

By letting $z \rightarrow \overline{1}$ through real values, we have

$$
\begin{aligned}
& \sum_{k=1}^{+\infty} \prod_{i=1}^{n}[k-i]_{q}\left((k-n-1)^{2}+\lambda \beta\right) a_{k} \\
& \leq \frac{\beta(1+\lambda)\left((-1)^{n-1} \prod_{k=1}^{n}\left(1+q+\cdots+q^{k-1}\right)-\alpha\right)}{q^{\left(\begin{array}{l}
n+1 \\
n-1
\end{array}\right)}} .
\end{aligned}
$$


Conversely, Let equation 2.1 holds true, by equation 1.6 it is enough to show that

$$
\begin{aligned}
& X(f)= \\
& \left|\frac{z^{n+3}\left(D_{q}^{n} f(z)\right)^{\prime \prime}+z^{n+2}\left(D_{q}^{n} f(z)\right)^{\prime}-\frac{(-1)^{n}(n+1)^{2}}{q^{\left(\begin{array}{c}
n+1 \\
n-1
\end{array}\right)}} \prod_{k=1}^{n}\left(1+q+\cdots+q^{k-1}\right)}{\lambda z^{n+1}\left(D_{q}^{n} f(z)\right)+\frac{(-1)^{n} \prod_{k=1}^{n}\left(1+q+\cdots+q^{k-1}\right)}{q^{\left(\begin{array}{c}
n+1 \\
n-1
\end{array}\right)}}+\frac{(1+\lambda) \alpha}{q^{\left(\begin{array}{c}
n+1 \\
n-1
\end{array}\right)}}}\right| \\
& <\beta,
\end{aligned}
$$

or

$$
\begin{aligned}
& \quad X(f)= \\
& \quad\left|z^{n+3}\left(D_{q}^{n} f(z)\right)^{\prime \prime}+z^{n+2}\left(D_{q}^{n} f(z)\right)^{\prime}-\frac{(-1)^{n}(n+1)^{2}}{q^{\left(\begin{array}{c}
n+1 \\
n-1
\end{array}\right)}} \prod_{k=1}^{n}\left(1+q+\cdots+q^{k-1}\right)\right| \\
& -\quad \beta\left|\lambda z^{n+1}\left(D_{q}^{n} f(z)\right)+\frac{(-1)^{n} \prod_{k=1}^{n}\left(1+q+\cdots+q^{k-1}\right)}{q^{\left(\begin{array}{c}
n+1 \\
n-1
\end{array}\right)}}+\frac{(1+\lambda) \alpha}{q^{\left(\begin{array}{c}
n+1 \\
n-1
\end{array}\right)}}\right| \\
& <\quad 0 .
\end{aligned}
$$

But for $0<|z|=r<1$ we have

$$
\begin{aligned}
X(f) & =\left|\sum_{k=1}^{+\infty} \prod_{i=1}^{n}[k-i]_{q}(k-n-1)^{2} a_{k} z^{k}\right| \\
& -\beta \mid \frac{(1+\lambda)}{q^{\left(\begin{array}{c}
n+1 \\
n-1
\end{array}\right)}}\left((-1)^{n-1} \prod_{k=1}^{n}\left(1+q+q^{2}+\cdots+q^{k-1}\right)-\alpha\right) \\
& -\lambda \sum_{k=1}^{+\infty} \prod_{i=1}^{n}[k-i]_{q} a_{k} z^{k} \mid \\
& \leq \sum_{k=1}^{+\infty} \prod_{i=1}^{n}[k-i]_{q}(k-n-1)^{2}\left|a_{k}\right| r^{k} \\
& -\frac{\beta(1+\lambda)\left((-1)^{n-1} \prod_{k=1}^{n}\left(1+q+\cdots+q^{k-1}\right)-\alpha\right)}{q^{\left(\begin{array}{c}
n+1 \\
n-1
\end{array}\right)}} \\
& +\lambda \beta \sum_{k=1}^{+\infty} \prod_{i=1}^{n}[k-i]_{q}\left|a_{k}\right| r^{k} \leq \sum_{k=1}^{+\infty} \prod_{i=1}^{n}[k-i]_{q}\left((k-n-1)^{2}-\lambda \beta\right)\left|a_{k}\right| r^{k} \\
& -\frac{\left.\beta(1+\lambda)\left((-1)^{n-1} \prod_{k=1}^{n}\left(1+q+\cdots+q^{k-1}\right)-\alpha\right)\right)}{q^{\left(\begin{array}{c}
n+1 \\
n-1
\end{array}\right)} .}
\end{aligned}
$$

Since the above inequality holds for all $r(0<r<1)$, by letting $r \rightarrow \overline{1}$ and using equation 2.1 we obtain $X(f) \leq 0$, and this completes the proof. 
Corollary 2.1. If function $f(z)$ of the form equation 1.1 belongs to $\sum_{q}(n ; \lambda, \alpha, \beta)$ then

$$
a_{k} \leq \frac{\beta(1+\lambda)\left((-1)^{n-1} \prod_{k=1}^{n}\left(1+q+\cdots+q^{k-1}\right)-\alpha\right)}{q^{\left(\begin{array}{c}
n+1 \\
n-1
\end{array}\right)} \prod_{i=1}^{n}[k-i]_{q}\left((k-n-1)^{2}+\lambda \beta\right)} .
$$

This result is sharp for $H(z)$ given by

$$
H(z)=\frac{1}{z}+\frac{\beta(1+\lambda)\left((-1)^{n-1} \prod_{k=1}^{n}\left(1+q+\cdots+q^{k-1}\right)-\alpha\right)}{q^{\left(\begin{array}{c}
n+1 \\
n-1
\end{array}\right)} \prod_{i=1}^{n}[k-i]_{q}\left((k-n-1)^{2}+\lambda \beta\right)} z^{k-1} .
$$

Next we obtain extreme points and convex linear combination property for $f(z)$ belongs to $\sum_{q}(n ; \lambda, \alpha, \beta)$.

Theorem 2.2. The function $f(z)$ of the form equation 1.1 belongs to $\sum_{q}(n ; \lambda, \alpha, \beta)$ if and only if it can be expressed by $f(z)=\sum_{k=0}^{\infty} \sigma_{k} f_{k}(z), \sigma_{k} \geq 0, \sum_{k=0}^{\infty} \sigma_{k}=1$ where $f_{0}(z)=\frac{1}{z}$ and

$f_{k}(z)=\frac{1}{z}+\frac{\beta(1+\lambda)\left((-1)^{n-1} \prod_{k=1}^{n}\left(1+q+\cdots+q^{k-1}\right)-\alpha\right)}{q^{(n+1)} \prod_{i=1}^{n}[k-i]_{q}\left((k-n-1)^{2}+\lambda \beta\right)} z^{k-1},(k=1,2, \ldots)$.

Proof. Let

$$
\begin{aligned}
f(z) & =\sum_{k=0}^{\infty} \sigma_{k} f_{k}(z) \\
& =\sigma_{0} f_{0}(z) \\
& +\sum_{k=1}^{\infty} \sigma_{k}\left[\frac{1}{z}+\frac{\beta(1+\lambda)\left((-1)^{n-1} \prod_{k=1}^{n}\left(1+q+\cdots+q^{k-1}\right)-\alpha\right)}{q^{\left(\begin{array}{c}
n+1 \\
n-1
\end{array}\right)} \prod_{i=1}^{n}[k-i]_{q}\left((k-n-1)^{2}+\lambda \beta\right)} z^{k-1}\right] \\
& =\frac{1}{z}+\sum_{k=1}^{\infty} \frac{\beta(1+\lambda)\left((-1)^{n-1} \prod_{k=1}^{n}\left(1+q+\cdots+q^{k-1}\right)-\alpha\right)}{q^{\left(\begin{array}{l}
n+1 \\
n-1
\end{array}\right)} \prod_{i=1}^{n}[k-i]_{q}\left((k-n-1)^{2}+\lambda \beta\right)} \sigma_{k} z^{k-1} .
\end{aligned}
$$

Now by using Theorem 2.1 we conclude that $f(z) \in \sum_{q}(n ; \lambda, \alpha, \beta)$.

Conversely, if $f(z)$ given by equation 1.1 belongs to $\sum_{q}(n ; \lambda, \alpha, \beta)$, by letting $\sigma_{0}=1-\sum_{k=1}^{+\infty} \sigma_{k}$, where

$$
\sigma_{k}=\frac{q^{\left(\begin{array}{c}
n+1 \\
n-1
\end{array}\right)} \prod_{i=1}^{n}[k-i]_{q}\left((k-n-1)^{2}+\lambda \beta\right)}{\beta(1+\lambda)\left((-1)^{n-1} \prod_{k=1}^{n}\left(1+q+q^{2}+\cdots+q^{k-1}\right)-\alpha\right)} a_{k}, \quad(k=1,2, \ldots) .
$$

we conclude the required result.

Theorem 2.3. Let for $n=1,2, \ldots, m, f_{n}(z)=\frac{1}{z}+\sum_{k=1}^{+\infty} a_{k, n} z^{k-1}$ belongs to $\sum_{q}(n ; \lambda, \alpha, \beta)$, then $F(z)=\sum_{n=1}^{m} \sigma_{n} f_{n}(z)$ is also in the same class, where $\sum_{n=1}^{m} \sigma_{n}=1$. (Hence $\sum_{q}(n ; \lambda, \alpha, \beta)$ is a convex set. ) 
Proof. According to Theorem 2.1 for every $n=1,2, \ldots, m$ we have

$$
\begin{aligned}
& \sum_{k=1}^{+\infty} \prod_{i=1}^{n}[k-i]_{q}\left((k-n-1)^{2}+\lambda \beta\right) a_{k, n} \\
& \leq \frac{\beta(1+\lambda)\left((-1)^{n-1} \prod_{k=1}^{n}\left(1+q+q^{2}+\cdots+q^{k-1}\right)-\alpha\right)}{q^{\left(\begin{array}{l}
n+1 \\
n-1
\end{array}\right)}} .
\end{aligned}
$$

But

$$
\begin{aligned}
F(z) & =\sum_{n=1}^{m} \sigma_{n} f_{n}(z) \\
& =\sum_{n=1}^{m} \sigma_{n}\left(\frac{1}{z}+\sum_{k=1}^{\infty} a_{k, n} z^{k-1}\right) \\
& =\frac{1}{z} \sum_{n=1}^{m} \sigma_{n}+\sum_{k=1}^{\infty}\left(\sum_{n=1}^{m} \sigma_{n} a_{k, n}\right) z^{k-1} \\
& =\frac{1}{z}+\sum_{k=1}^{\infty}\left(\sum_{n=1}^{m} \sigma_{n} a_{k, n}\right) z^{k-1} .
\end{aligned}
$$

Since :

$$
\begin{aligned}
& \sum_{k=1}^{+\infty} \prod_{i=1}^{n}[k-i]_{q}\left((k-n-1)^{2}+\lambda \beta\right)\left(\sum_{n=1}^{m} \sigma_{n} a_{k, n}\right) \\
& =\sum_{n=1}^{m} \sigma_{n}\left(\sum_{k=1}^{+\infty} \prod_{i=1}^{n}[k-i]_{q}\left((k-n-1)^{2}+\lambda \beta\right)\right) a_{k, n} \\
& \leq \sum_{n=1}^{m} \sigma_{n} \frac{\beta(1+\lambda)\left((-1)^{n-1} \prod_{k=1}^{n}\left(1+q+q^{2}+\cdots+q^{k-1}\right)-\alpha\right)}{q^{\left(\begin{array}{c}
n+1 \\
n-1
\end{array}\right)}} \\
& =\frac{\beta(1+\lambda)\left((-1)^{n-1} \prod_{k=1}^{n}\left(1+q+q^{2}+\cdots+q^{k-1}\right)-\alpha\right)}{q^{\left(\begin{array}{c}
n+1 \\
n-1
\end{array}\right)}} \sum_{n=1}^{m} \sigma_{n} \\
& =\frac{\beta(1+\lambda)\left((-1)^{n-1} \prod_{k=1}^{n}\left(1+q+q^{2}+\cdots+q^{k-1}\right)-\alpha\right)}{q^{\left(\begin{array}{l}
n+1 \\
n-1
\end{array}\right)}}
\end{aligned}
$$

then by Theorem 2.1 the proof is complete.

\section{Radii condition and partial sum property}

In this section we obtain radii of starlikeness and convexity and investigate about partial sum property. 
Theorem 3.1. if the function $f(z)$ defined by equation 1.1 is in the class $\sum_{q}(n ; \lambda, \alpha, \beta)$, then $f(z)$ is meromorphically univalent starlike of order $\gamma$ in disk $|z|<R_{1}$, and it is meromorphically univalent convex of order $\gamma$ in disk $|z|<R_{2}$ where

$$
\begin{aligned}
& R_{1}=\inf _{k}\left\{\frac{q^{\left(\begin{array}{c}
n+1 \\
n-1
\end{array}\right)} \prod_{i=1}^{n}[k-i]_{q}\left((k-n-1)^{2}+\lambda \beta\right)(1-\gamma)}{\beta(1+\lambda)\left((-1)^{n-1} \prod_{k=1}^{n}\left(1+q+q^{2}+\cdots+q^{k-1}\right)-\alpha\right)(k+1+\gamma)}\right\}^{\frac{1}{k}} \\
& R_{2}=\inf _{k}\left\{\frac{q^{\left(\begin{array}{c}
n+1 \\
n-1
\end{array}\right)} \prod_{i=1}^{n}[k-i]_{q}\left((k-n-1)^{2}+\lambda \beta\right)(1-\gamma)}{\beta(k-1)(1+\lambda)\left((-1)^{n-1} \prod_{k=1}^{n}\left(1+q+q^{2}+\cdots+q^{k-1}\right)-\alpha\right)(k+1+\gamma)}\right\}^{\frac{1}{k}}
\end{aligned}
$$

Proof. For starlikeness it is enough to show that

$$
\left|\frac{z f(z)^{\prime}+f(z)}{f(z)}\right|<1-\gamma
$$

but

$$
\left|\frac{z f(z)^{\prime}+f(z)}{f(z)}\right|=\left|\frac{\sum_{k=1}^{+\infty} k a_{k} z^{k}}{1+\sum_{k=1}^{+\infty} a_{k} z^{k}}\right| \leq \frac{\sum_{k=1}^{+\infty} k a_{k}|z|^{k}}{1-\sum_{k=1}^{+\infty} a_{k}|z|^{k}} \leq 1-\gamma
$$

or

$$
\sum_{k=1}^{+\infty} k a_{k}|z|^{k} \leq(1-\gamma)-(1-\gamma) \sum_{k=1}^{+\infty} a_{k}|z|^{k}
$$

or

$$
\sum_{k=1}^{+\infty} \frac{k+1-\gamma}{1-\gamma} a_{k}|z|^{k} \leq 1
$$

By using equation 2.1 and equation 3.3 we obtain

$$
\frac{k+1-\gamma}{1-\gamma}|z|^{k} \leq \frac{\beta(1+\lambda)\left((-1)^{n-1} \prod_{k=1}^{n}\left(1+q+q^{2}+\cdots+q^{k-1}\right)-\alpha\right)}{q^{\left(\begin{array}{c}
n+1 \\
n-1
\end{array}\right)} \prod_{i=1}^{n}[k-i]_{q}\left((k-n-1)^{2}+\lambda \beta\right)} .
$$

So, it is enough to suppose

$$
|z|^{k} \leq \frac{q^{\left(\begin{array}{c}
n+1 \\
n-1
\end{array}\right)} \prod_{i=1}^{n}[k-i]_{q}\left((k-n-1)^{2}+\lambda \beta\right)(1-\gamma)}{\beta(1+\lambda)\left((-1)^{n-1} \prod_{k=1}^{n}\left(1+q+q^{2}+\cdots+q^{k-1}\right)-\alpha\right)(k+1-\gamma)} .
$$

Hence we get the required result equation 3.1. For convexity, by using the Alexander,s Theorem(If $f$ be an analytic function in the unit disk and normalized by $f(0)=f^{\prime}(0)-1=0$, then $f(z)$ is convex if and only if $z f^{\prime}(z)$ is starlike.) and applying an easy calculation we conclude the required result equation 3.2. So the proof is complete. 
Theorem 3.2. Let $f(z) \in \sum$, and define

$$
S_{1}(z)=\frac{1}{z} \quad, \quad S_{m}(z)=\frac{1}{z}+\sum_{k=1}^{m-1} a_{k} z^{k-1} \quad, \quad(m=2,3, \ldots) .
$$

Also suppose $\sum_{k=1}^{+\infty} x_{k} a_{k} \leq 1$, where

$$
x_{k}=\frac{q^{\left(\begin{array}{c}
n+1 \\
n-1
\end{array}\right)} \prod_{i=1}^{n}[k-i]_{q}\left((k-n-1)^{2}+\lambda \beta\right)}{\beta(1+\lambda)\left((-1)^{n-1} \prod_{k=1}^{n}\left(1+q+q^{2}+\cdots+q^{k-1}\right)-\alpha\right)},
$$

then

$$
\operatorname{Re}\left(\frac{f(z)}{S_{m}(z)}\right)>1-\frac{1}{x_{m}} \quad, \quad \operatorname{Re}\left(\frac{S_{m}(z)}{f(z)}\right)>\frac{x_{m}}{1+x_{m}}
$$

Proof. Since $\sum_{k=1}^{+\infty} x_{k} a_{k} \leq 1$, they by Theorem 2.1, $f(z) \in \sum_{q}(n ; \lambda, \alpha, \beta)$. Also by equation 1.4 and equation 1.5 we have

SO

$$
\frac{\prod_{i=1}^{n}[k-i]_{q}}{(-1)^{n-1} \prod_{k=1}^{n}\left(1+q+q^{2}+\cdots+q^{k-1}\right)-\alpha} \geq 1
$$

$$
x_{k}>\frac{q^{\left(\begin{array}{c}
n+1 \\
n-1
\end{array}\right)}\left((k-n-1)^{2}+\lambda \beta\right)}{\beta(1+\lambda)},
$$

and $\left\{x_{k}\right\}$ is an increasing sequence, therefore we obtain

$$
\sum_{k=1}^{m-1} a_{k}+x_{m} \sum_{k=m}^{+\infty} a_{k} \leq 1 .
$$

Now by putting

$$
X(z)=x_{m}\left[\frac{f(z)}{S_{m}(z)}-\left(1-\frac{1}{x_{m}}\right)\right],
$$

and making use of equation 3.7 we obtain

$$
\operatorname{Re}\left(\frac{X(z)-1}{X(z)+1}\right) \leq\left|\frac{X(z)-1}{X(z)+1}\right|=\left|\frac{x_{m} f(z)-x_{m} S_{m}(z)}{x_{m} f(z)-x_{m} S_{m}(z)+2 S_{m}(z)}\right|
$$

By a simple calculation we get $\operatorname{Re}(X(z))>0$, therefore $\operatorname{Re}\left(\frac{X(z)}{x_{m}}\right)>0$, or equivalently $\operatorname{Re}\left[\frac{f(z)}{S_{m}(z)}-\left(1-\frac{1}{x_{m}}\right)\right]>0$, and this gives the first inequality in equation 3.6. For the second inequality we consider

$$
Y(z)=\left(1+x_{m}\right)\left[\frac{S_{m}(z)}{f(z)}-\frac{x_{m}}{1+x_{m}}\right]
$$

and by using equation 3.7 we have $\left|\frac{Y(z)-1}{Y(z)+1}\right| \leq 1$, and Hence $\operatorname{Re}(Y(z))>0$, therefore $\operatorname{Re}\left(\frac{Y(z)}{1+x_{m}}\right)>0$, or equivalently $\operatorname{Re}\left[\frac{S_{m}(z)}{f(z)}-\frac{x_{m}}{1+x_{m}}\right]>0$, and this shows the second inequality in equation 3.6. So the proof is complete. 


\section{Some properties of $\sum_{q}(n ; \lambda, \alpha, \beta)$}

Theorem 4.1. Let $f(z), g(z) \in \sum_{q}(n ; \lambda, \alpha, \beta)$ and given by $f(z)=\frac{1}{z}+\sum_{k=1}^{+\infty} a_{k} z^{k-1}, g(z)=\frac{1}{z}+\sum_{k=1}^{+\infty} b_{k} z^{k-1}$. Then the function $h(z)=\frac{\tilde{1}}{z}+\sum_{k=1}^{+\infty}\left(a_{k}^{2}+b_{k}^{2}\right) z^{k-1}$ is also in $\sum_{q}(n ; \gamma, \alpha, \beta)$ where $\gamma \leq \frac{\lambda}{2}-\frac{(k-n-1)^{2}}{2 \beta}$.

Proof. Since $f(z), g(z) \in \sum_{q}(n ; \lambda, \alpha, \beta)$ therefore we have

$$
\begin{aligned}
& \sum_{k=1}^{\infty}\left[\prod_{i=1}^{n}[k-i]_{q}\left((k-n-1)^{2}+\lambda \beta\right)\right]^{2} a_{k}^{2} \\
\leq & {\left[\sum_{k=1}^{+\infty} \prod_{i=1}^{n}[k-i]_{q}\left((k-n-1)^{2}+\lambda \beta\right) a_{k}\right]^{2} } \\
\leq & {\left[\frac{\beta(1+\lambda)\left((-1)^{n-1} \prod_{k=1}^{n}\left(1+q+q^{2}+\cdots+q^{k-1}\right)-\alpha\right)}{q^{\left(\begin{array}{l}
n+1 \\
n-1
\end{array}\right)}}\right]^{2} }
\end{aligned}
$$

and

$$
\begin{aligned}
& \sum_{k=1}^{+\infty}\left[\prod_{i=1}^{n}[k-i]_{q}\left((k-n-1)^{2}+\lambda \beta\right)\right]^{2} b_{k}^{2} \\
\leq & {\left[\sum_{k=1}^{+\infty} \prod_{i=1}^{n}[k-i]_{q}\left((k-n-1)^{2}+\lambda \beta\right) b_{k}\right]^{2} } \\
\leq & {\left[\frac{\beta(1+\lambda)\left((-1)^{n-1} \prod_{k=1}^{n}\left(1+q+q^{2}+\cdots+q^{k-1}\right)-\alpha\right)}{q^{\left(\begin{array}{l}
n+1 \\
n-1
\end{array}\right)}}\right]^{2} . }
\end{aligned}
$$

The above inequalities yield us

$$
\begin{aligned}
& \sum_{k=1}^{\infty} \frac{1}{2}\left[\prod_{i=1}^{n}[k-i]_{q}\left((k-n-1)^{2}+\lambda \beta\right)\right]^{2}\left(a_{k}^{2}+b_{k}^{2}\right) \\
& \leq\left[\frac{\beta(1+\lambda)\left((-1)^{n-1} \prod_{k=1}^{n}\left(1+q+q^{2}+\cdots+q^{k-1}\right)-\alpha\right)}{q^{\left(\begin{array}{l}
n+1 \\
n-1
\end{array}\right)}}\right]^{2}
\end{aligned}
$$

Now we must show

$$
\begin{aligned}
& \sum_{k=1}^{\infty}\left[\prod_{i=1}^{n}[k-i]_{q}\left((k-n-1)^{2}+\gamma \beta\right)\right]^{2}\left(a_{k}^{2}+b_{k}^{2}\right) \\
& \leq\left[\frac{\beta(1+\lambda)\left((-1)^{n-1} \prod_{k=1}^{n}\left(1+q+q^{2}+\cdots+q^{k-1}\right)-\alpha\right)}{q^{\left(\begin{array}{l}
n+1 \\
n-1
\end{array}\right)}}\right]^{2}
\end{aligned}
$$

But above inequalities holds if 


$$
\prod_{i=1}^{n}[k-i]_{q}\left((k-n-1)^{2}+\gamma \beta\right) \leq \frac{1}{2}\left[\prod_{i=1}^{n}[k-i]_{q}\left((k-n-1)^{2}+\lambda \beta\right)\right]
$$

or equivalently

$$
2(k-n-1)^{2}+2 \gamma \beta \leq(k-n-1)^{2}+\lambda \beta
$$

or

$$
\gamma \leq \frac{\lambda}{2}-\frac{(k-n-1)^{2}}{2 \beta}
$$

Theorem 4.2. The class $\sum_{q}(n ; \lambda, \alpha, \beta)$ is a convex set.

Proof. Let

$$
f(z)=\frac{1}{z}+\sum_{k=1}^{\infty} a_{k} z^{k-1}
$$

and

$$
g(z)=\frac{1}{z}+\sum_{k=1}^{\infty} b_{k} z^{k-1}
$$

be in the class $\sum_{q}(n ; \lambda, \alpha, \beta)$. For $t \in(0,1)$, it is enough to show that the function $h(z)=(1-t) f(z)+t g(z)$ is in the class $\sum_{q}(n ; \lambda, \alpha, \beta)$. Since

$$
h(z)=\frac{1}{z}+\sum_{k=1}^{\infty}\left((1-t) a_{k}+t b_{k}\right) z^{k-1}
$$

then

$$
\begin{aligned}
& \sum_{k=1}^{\infty}\left[\prod_{i=1}^{n}[k-i]_{q}\left((k-n-1)^{2}+\lambda \beta\right)\right]\left((1-t) a_{k}+t b_{k}\right) \\
& \leq \frac{\beta(1+\lambda)\left((-1)^{n-1} \prod_{k=1}^{n}\left(1+q+q^{2}+\cdots+q^{k-1}\right)-\alpha\right)}{\left.q^{\left(_{n-1}^{n+1}\right.}\right)}
\end{aligned}
$$

so $h(z) \in \sum_{q}(n ; \lambda, \alpha, \beta)$.

Corollary 4.1. Let $f_{j}(z) \quad(j=1,2, \ldots, n)$, defined by $f_{j}(z)=\frac{1}{z}+\sum_{k=1}^{\infty} a_{k, j} z^{k-1}$ be in the class $\sum_{q}(n ; \lambda, \alpha, \beta)$, then the function $F(z)=\sum_{j=1}^{n} c_{j} f_{j}(z)$ is also in $\sum_{q}(n ; \lambda, \alpha, \beta)$ where $\sum_{j=1}^{n} c_{j}=1$. 


\section{Hadamard product}

For the functions $f(z), g(z) \in \Sigma$ is given by equation 1.1, we denote by $(f * g)(z)$ the Hadamard product (or convolution) of the functions $f(z), g(z)$, that is

$$
(f * g)(z)=\frac{1}{z}+\sum_{k=1}^{+\infty} a_{k} b_{k} z^{k-1}=(g * f)(z)
$$

Theorem 5.1. If $f(z), g(z)$ defined by equation 1.1 is in the class $\sum_{q}(n ; \lambda, \alpha, \beta)$ then $(f * g)(z)=\frac{1}{z}+\sum_{k=1}^{+\infty} a_{k} b_{k} z^{k-1}$ in the class $\sum_{q}(n ; \gamma, \alpha, \beta)$ where

$$
\gamma \leq \frac{q^{\left(\begin{array}{c}
n+1 \\
n-1
\end{array}\right)} \prod_{i=1}^{n}[k-i]_{q}\left((k-n-1)^{2}+\lambda \beta\right)^{2}}{\beta^{2}(1+\lambda)\left((-1)^{n-1} \prod_{k=1}^{n}\left(1+q+q^{2}+\cdots+q^{k-1}\right)-\alpha\right)}-\frac{(k-n-1)^{2}}{\beta} .
$$

Proof. Since $f(z), g(z) \in \sum_{q}(n ; \lambda, \alpha, \beta)$, so by equation 2.1

$\sum_{k=1}^{\infty} \prod_{i=1}^{n}[k-i]_{q}\left((k-n-1)^{2}+\lambda \beta\right) a_{k} \leq \frac{\beta(1+\lambda)\left((-1)^{n-1} \prod_{k=1}^{n}\left(1+q+\cdots+q^{k-1}\right)-\alpha\right)}{q^{\left(\begin{array}{c}n+1 \\ n-1\end{array}\right)}}$

and

$$
\begin{aligned}
& \sum_{k=1}^{\infty} \prod_{i=1}^{n}[k-i]_{q}\left((k-n-1)^{2}+\lambda \beta\right) b_{k} \\
& \leq \frac{\beta(1+\lambda)\left((-1)^{n-1} \prod_{k=1}^{n}\left(1+q+\cdots+q^{k-1}\right)-\alpha\right)}{q^{\left(\begin{array}{c}
n+1 \\
n-1
\end{array}\right)}}
\end{aligned}
$$

By using the equation 5.1, equation 5.2 and Cauchy-Schwarts inequality we have

$$
\begin{aligned}
& \sum_{k=1}^{\infty} \prod_{i=1}^{n}[k-i]_{q}\left((k-n-1)^{2}+\lambda \beta\right) \sqrt{a_{k} b_{k}} \\
& \leq \frac{\beta(1+\lambda)\left((-1)^{n-1} \prod_{k=1}^{n}\left(1+q+\cdots+q^{k-1}\right)-\alpha\right)}{q^{\left(\begin{array}{c}
n+1 \\
n-1
\end{array}\right)}} .
\end{aligned}
$$

we must find the smallest $\gamma$ such that

$$
\begin{aligned}
& \sum_{k=1}^{\infty} \prod_{i=1}^{n}[k-i]_{q}\left((k-n-1)^{2}+\gamma \beta\right) a_{k} b_{k} \\
& \leq \frac{\beta(1+\lambda)\left((-1)^{n-1} \prod_{k=1}^{n}\left(1+q+\cdots+q^{k-1}\right)-\alpha\right)}{q^{\left(\begin{array}{l}
n+1 \\
n-1
\end{array}\right)}} .
\end{aligned}
$$


Now it is enough to show that

$$
\begin{aligned}
& \prod_{i=1}^{n}[k-i]_{q}\left((k-n-1)^{2}+\gamma \beta\right) a_{k} b_{k} \\
& \leq \prod_{i=1}^{n}[k-i]_{q}\left((k-n-1)^{2}+\lambda \beta\right) \sqrt{a_{k} b_{k}}
\end{aligned}
$$

or equivalently

$$
\sqrt{a_{k} b_{k}} \leq \frac{(k-n-1)^{2}+\lambda \beta}{(k-n-1)^{2}+\gamma \beta}
$$

But from equation 5.3,

$$
\sqrt{a_{k} b_{k}} \leq \frac{\beta(1+\lambda)\left((-1)^{n-1}\left(\prod_{k=1}^{n}\left(1+q+q^{2}+\cdots+q^{k-1}\right)-\alpha\right)\right.}{q^{\left(\begin{array}{c}
n+1 \\
n-1
\end{array}\right)} \prod_{i=1}^{n}[k-i]_{q}\left((k-n-1)^{2}+\lambda \beta\right)}
$$

so it is enough that

$$
\begin{aligned}
& \frac{\beta(1+\lambda)\left((-1)^{n-1} \prod_{k=1}^{n}\left(1+q+q^{2}+\cdots+q^{k-1}\right)-\alpha\right)}{q^{\left(\begin{array}{c}
n+1 \\
n-1
\end{array}\right)} \prod_{i=1}^{n}[k-i]_{q}\left((k-n-1)^{2}+\lambda \beta\right)} \\
& \leq \frac{(k-n-1)^{2}+\lambda \beta}{(k-n-1)^{2}+\gamma \beta}
\end{aligned}
$$

By using the equation 5.6 we have

$$
\begin{aligned}
\gamma & \leq \frac{q^{\left(\begin{array}{c}
n+1 \\
n-1
\end{array}\right)} \prod_{i=1}^{n}[k-i]_{q}\left((k-n-1)^{2}+\lambda \beta\right)^{2}}{\beta^{2}(1+\lambda)\left((-1)^{n-1} \prod_{k=1}^{n}\left(1+q+q^{2}+\cdots+q^{k-1}\right)-\alpha\right)} \\
& -\frac{(k-n-1)^{2}}{\beta} .
\end{aligned}
$$

\section{R E F E R E N C E S}

1. M. H. Abu-Risha, M. H. Annaby, M. E. H. Ismail and Z. S. Mansour: Linear q-difference equations, Z. Anal. Anwend. 26 (4) (2007), 481-494.

2. B. Ahmad and M. ARIF: New subfamily of meromorphic convex functions in circular domain involving q-operators, Int. J. Anal. Appl. 1 (2018), 75-82.

3. H. Aldweby and M. DARUs: A subclass of harmonic univalent functions associated with q-analogue of Dziok Srivastava operator, ISRN Math. Anal. 6 (2013), 382-312.

4. D. Albayrak, S. D. Purohit and F. UCAR: On q-analogues of sumudu transforms, JAn. Stiint. Univ. Ovidius Constanta Ser. Mat. 21 (1) (2013), 239-260. 
5. G. BAngerezako: Variational calculus on q-nonuniform lattices, J. Math. anal. Appl. 306 (2005), no. 161-179.

6. T. ERnst: A comprehensive treatment of q-calculus, JBirkhauser/Springer Basel AG, Basel, 2012.

7. G. Gasper and M. Rahman: Basic Hypergeometric series, Cambridge University Press, Cambridge, 1990.

8. F. H. JACKSON: On q-functions and a certain difference operator, Trans. Royal Soc. Edinburgh, 46(2) (1909), 253-281.

9. V. Kasand P. Cheung: Quantum calclus, Universitext, Springer, New York, 2002.

10. Z. S. I. MANSOUR: Linear sequential q-difference equations of fractional order, Fract. Calc. Appl. Anal. 12(2) (2009), 159-178.

11. A. O. Mostafa, M. K. Aouf, H. M. Zayed and T. Bulboaca: Convolution conditions for subelasse of mermorphic functions of complex order associated with basic Bessel functions, J. Egyptian Math. Soc. 25 (2017), 286-290.

12. H. E. OzKan UCAR: coefficient inequalties for q-starlike functions, Appl. Math. Comput. 276 (2016), 122--126.

13. Y. Polatoglu: Growth and distortion theorems for generalized q-starlike functions, Adv. Math. Sci. J. 5 (2016), 7--12.

14. S. D. Purohit and R. K. Raina: Certain subclass of analytic functions associated with fractional q-calculus operators, Math. Scand. 109 (2011), 55--70.

15. S. D. PUronit: textitA new class of multivalently analytic functions associated with fractional $q$-calculus operators, Fractional Differ. Calc. 2(2) (2012), 129-138.

16. P. M. Rajković, S. D. Marinković and M. S. Stanković: Fractional integrals and derivatives in q-calculus, Appl. Anal. Discrete Math., 1 (2007), 311-323.

17. T. M. SEOUdy and M. K. AOuF: Convolution properties for certain classes of analytic functions defined by q-derivative operator, Abster. Appl. Anal., 2014 (2014), Art. ID 846719, 1-7.

Mohammad Hassn Golmohammadi

Faculty of Mathematical Sciences

Department of Pure Mathematics

Payame Noor University, P. O. Bax: 19395 - 3697, Tehran, Iran,

golmohamadi@pnu.ac.ir

Shahram Najafzadeh

Faculty of Mathematical Sciences

Department of Pure Mathematics

Payame Noor University, P. O. Bax: 19395 - 3697, Tehran, Iran,

najafzadeh1234@yahoo.ie 
Mohammad Reza Foroutan

Faculty of Mathematical Sciences

Department of Pure Mathematics

Payame Noor University, P. O. Bax: 19395 - 3697, Tehran, Iran,

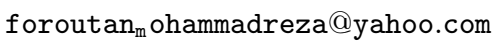

